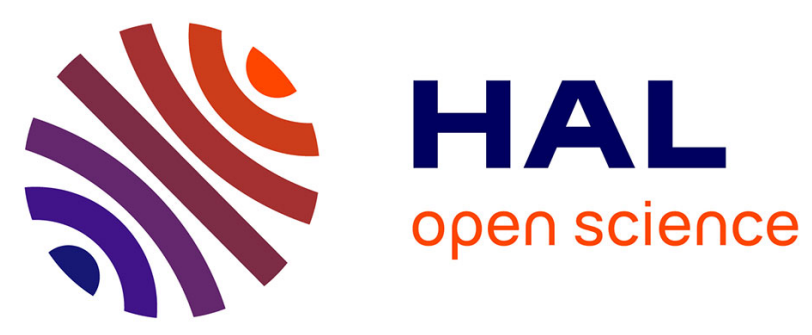

\title{
EXISTENCE DE DOMAINES ANGULAIRES DISJOINTS DE GÉNÉRATION DES ONDES RÉFRACTÉES DANS UN MATÉRIAU ANISOTROPE : NOUVELLE NOTION D'ANGLE CRITIQUE
}

H. Ribeiro, J. de Belleval, P. Lanceleur

\section{To cite this version:}

H. Ribeiro, J. de Belleval, P. Lanceleur. EXISTENCE DE DOMAINES ANGULAIRES DISJOINTS DE GÉNÉRATION DES ONDES RÉFRACTÉES DANS UN MATÉRIAU ANISOTROPE: NOUVELLE NOTION D'ANGLE CRITIQUE. Journal de Physique IV Proceedings, 1992, 02 (C1), pp.C1915-C1-918. 10.1051/jp4:19921200 . jpa-00251166

\author{
HAL Id: jpa-00251166 \\ https://hal.science/jpa-00251166
}

Submitted on 1 Jan 1992

HAL is a multi-disciplinary open access archive for the deposit and dissemination of scientific research documents, whether they are published or not. The documents may come from teaching and research institutions in France or abroad, or from public or private research centers.
L'archive ouverte pluridisciplinaire HAL, est destinée au dépôt et à la diffusion de documents scientifiques de niveau recherche, publiés ou non, émanant des établissements d'enseignement et de recherche français ou étrangers, des laboratoires publics ou privés. 


\title{
EXISTENCE DE DOMAINES ANGULAIRES DISJOINTS DE GENERATION DES ONDES RÉFRACTEES DANS UN MATÉRIAU ANISOTROPE : NOUVELLE NOTION D'ANGLE CRITIQUE
}

\author{
H. RIBEIRO, J.F. de BELLEVAL et P. LANCELEUR \\ UTC, LG2mS, BP. 649, F-60206 Compiègne cedex, France
}

\begin{abstract}
The problem of the reflection/transmission of monochomatic plane waves in oblique incidence is treated for two semi-infinite anisotropic media. The mathematical formalism used permits the determination of the amplitudes, polarisation and wave vectors as well as the energy propagation for all the possible cases including the evanescent or more generally inhomogeneous waves.
\end{abstract}

\section{1) INTRODUCTION}

L'utilisation de plus en plus importante par l'industrie de matériaux anisotropes, tels les composites ou certains aciers spéciaux, pose de nouveaux problèmes lors du contrôle non destructif par ultrasons. En effet, la nature de ces matériaux ne permet plus d'appliquer les lois de Snell-Descartes sous leur formalisme habituel pour la reflexion-réfraction des ondes aux interfaces entre matériaux. Le comportement complexe des faisceaux ultrasonores en pareils cas nécessite alors la prise en compte du caractère anisotrope du ou des milieux de propagation.

\section{2) ETUDE THEORIQUE}

\section{2-1 Introduction}

Le présent travail a pour objet l'étude des phénomènes de reflexion/transmission apparaissant lors de l'incidence d'une onde plane monochromatique quelconque dont on représente le déplacement par :

$$
\vec{u}(\vec{r}, t)=\vec{u}_{0} e^{i \varphi} \text { avec } \varphi=\vec{k} \cdot \vec{r}-\omega t, \vec{k} \text { etant le vecteur d'onde, }
$$

$\omega$ la pulsation, $\vec{r}$ et $t$ le rayon vecteur à l'origine et le temps respectivement, sur une interface plane séparant deux milieux anisotropes quelconques semi-infinis.

Partant des lois de Snell-Descartes qui établissent que tous les vecteurs d'ondes de toutes les ondes réfractées sont contenues dans le même plan, et que tous ces vecteurs admettent la même projection sur l'interface, on constate que le vecteur d'onde 
de chaque onde réfractée n'a plus qu'une seule inconnue : sa composante normale à l'interface. En utilisant le vecteur lenteur, défini comme l'inverse de la vitesse de phase selon sa direction de propagation, la détermination de cette composante inconnue revient à déterminer les points d'intersection de la droite normale à linterface passant par l'extrémité des composantes horizontales des vecteurs lenteur avec les traces des surfaces des lenteurs des ondes considérées dans le plan d'incidence [1] (Fig. 1).

Analytiquement, cela se traduit par la recherche de solutions non nulles des équations de dispersion d'une onde acoustique dans un milieu anisotrope de caractéristiques données.

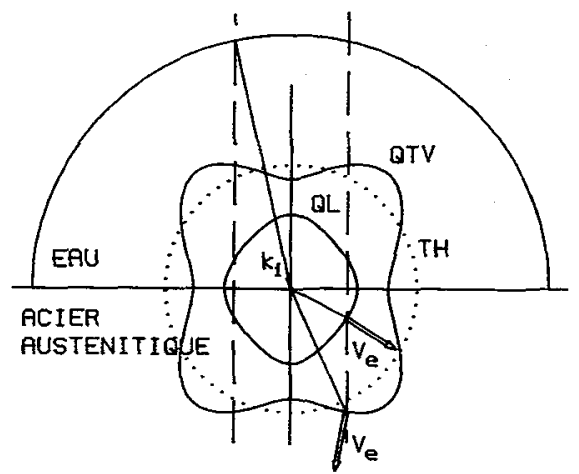

Figure 1 : direction de propagation et vitesse d'énergie $\left(V_{e}\right)$ des ondes transmises.

Cette équation, du sixième degré, admet pour racines les six composantes normales à l'interface de chacun des vecteurs lenteur des six ondes réfractées à l'interface [2]. Pour une onde et une incidence données, il se peut que certaines solutions ainsi trouvées soient complexes, traduisant par là-même l'absence d'intersection avec certaines traces des surfaces des lenteurs. Les vecteurs lenteurs correspondant à de telles racines seront également complexes, c'est à dire présentant un amortissement de l'onde suivant une direction donnée. Une telle onde sera dite inhomogène.

\section{2-2 Méthode employée}

Le formalisme numérique employé, qui permet la détermination des ondes ainsi créées, à savoir leurs directions de propagation ainsi que leurs amplitudes et polarisations, repose sur la résolution de l'équation aux valeurs propres suivante :

$$
\begin{aligned}
& G_{i k} P_{k}=0 \\
\text { où } & G_{i k}=C_{i j k l} m_{j} m_{1}-\rho \delta_{i k}
\end{aligned}
$$

avec $\vec{m}$ vecteur lenteur, $\rho$ masse volumique, $C_{i j k l}$ constantes élastiques du milieu de propagation, $\overline{\bar{\delta}}$ tenseur de Kronecker et $\overline{\mathbf{P}}$ vecteur polarisation de l'onde [3].

A la notion classique de polarisation et de vecteur d'onde réels, correspondant à des solutions réelles de l'équation ci-dessus, on doit ajouter le cas des polarisations et des vecteurs d'ondes complexes pour des solutions complexes de l'équation (Fig. 2) :

$$
\overrightarrow{\mathbf{P}}=\overrightarrow{\mathbf{P}}^{\prime}+\mathbf{i} \overrightarrow{\mathbf{P}}^{\prime \prime} \text { et } \overrightarrow{\mathbf{K}}=\overrightarrow{\mathbf{K}}^{\prime}+\mathbf{i} \vec{K}^{\prime \prime}
$$

C'est le cas des ondes inhomogènes décrites par ailleurs [4] dont la polarisation est elliptique [5]. On utilise dans ce cas le concept de bivecteur formalisé par Hayes [6].

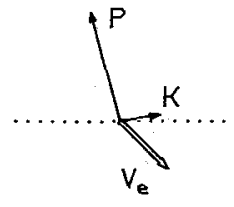

onde homogène

Figure 2 : différents types d'onde

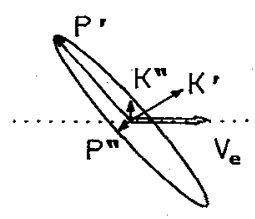

onde inhomogène 


\section{3) RES UITATS}

L'étude de quelques cas pratiques, comme la transmission eau-acier austénitique, permet la mise en évidence de plusieurs phénomènes remarquables.

Pour une onde incidente purement longitudinale, le milieu incident étant de l'eau, on a représenté les différents cas rencontrés (Fig. $3 a$ ) ainsi que l'évolution des vecteurs $\overrightarrow{\mathrm{P}}$ et $\vec{K}$ des ondes transmises dans le deuxième milieu (Fig. 3 b) en fonction de $l$ ' incidence $i . L^{\prime}$ onde incidente ayant sa polarisation ainsi que son vecteur d'onde entièrement contenus dans le plan de l'interface, il ne peut y avoir génération d'ondes de type transversale horizontale. Les deux types d'ondes générées dans ce cas seront donc quasi-longitudinal (QL) et quasi-transversal vertical (QTV).

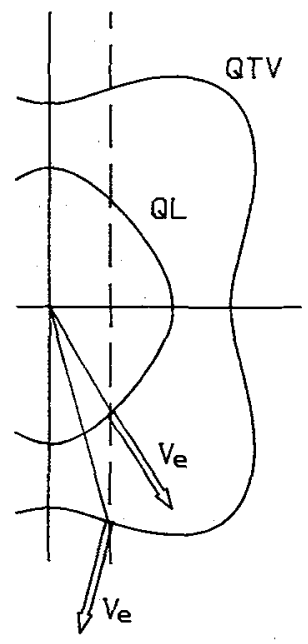

$\mathbf{i}<16^{\circ}$

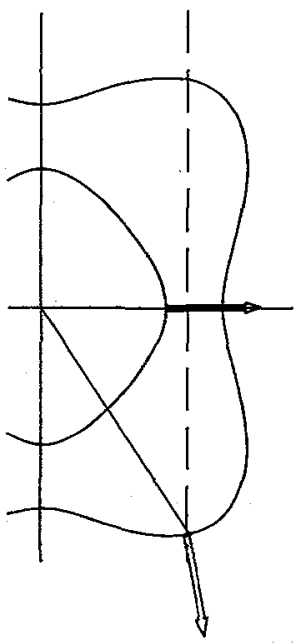

$16^{\circ}<\mathrm{i}<22^{\circ}$

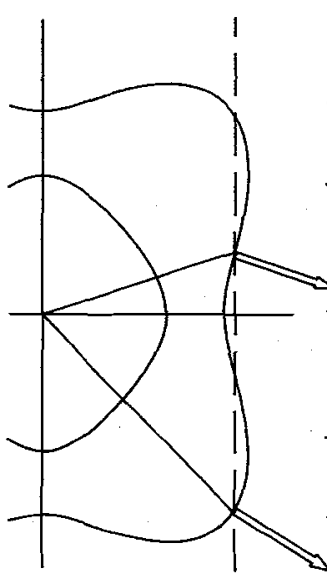

$23^{\circ}<\mathrm{i}<25^{\circ}$
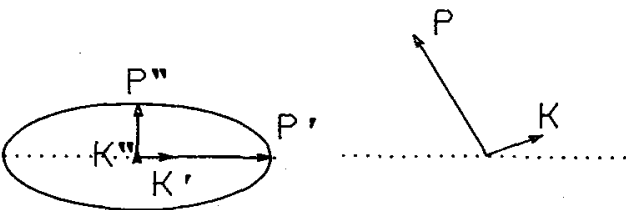

i $>25^{\circ}$

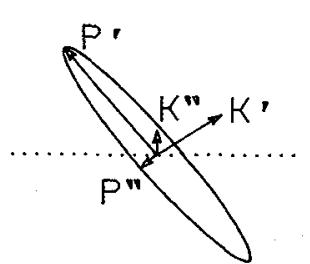

(b)
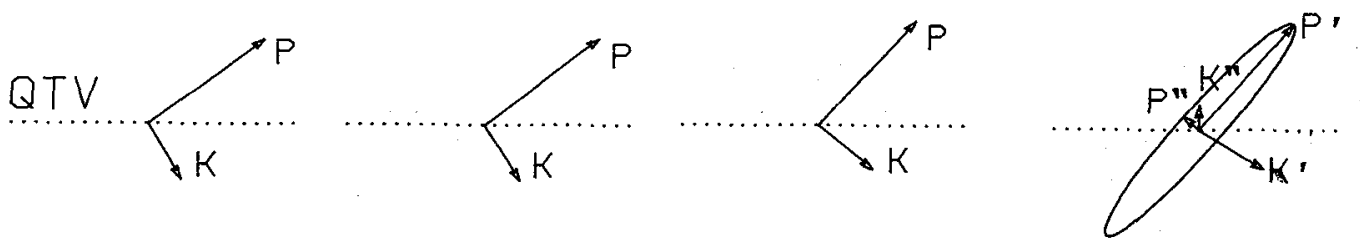

Figure 3 : évolution des ondes transmises avec l'angle d'incidence 
Lorsque i est inférieur à $15^{\circ}$, les 2 ondes générées sont propagatives. Entre $16^{\circ}$ et $22^{\circ}$, l'onde $\mathrm{QL}$, dont la trace de la surface des lenteurs n'est plus intersectée, devient inhomogène. On constate dans ce cas-là que $\vec{K}^{\prime} \perp \vec{K}^{\prime \prime}$ et $\vec{P}^{\prime} \perp \vec{P} "$. Entre $23^{\circ}$ et $25^{\circ}$, on assiste à un phénomène décrit précédemment [7-8], à savoir la réapparition d'onde. En effet pour cette plage angulaire, on obtient une double intersection de la surface des lenteurs QTV, il y a donc 2 ondes générées, dont une avec un angle de réfraction r $>90^{\circ}$, qui vont se propager dans le milieu. Enfin après $25^{\circ}$, les 2 ondes deviennent inhomogènes avec une vitesse d'énergie parallèle à l'interface. La combinaison de ces 2 ondes donne alors une onde évanescente comme dans les milieux isotropes au delà de l'incidence critique. Il convient de noter toutefois que cette combinaison n'est réalisée que dans certains cas particuliers, par exemple le cas où les traces des surfaces des lenteurs sont symétriques par rapport à l'interface.

La notion d'angle critique doit donc être reconsidérée [9] puisqu'il est possible, pour certaines configurations, d'avoir disparition de l'onde réfractée pour une plage angulaire limitée, puis réapparition de l'onde si l'on augmente l'incidence. Le critère retenu pour cette limite sera la direction de la vitesse d'ènergie. L'incidence critique sera définie comme l'angle pour lequel la direction de propagation de l'énergie devient parallèle à l'interface.

\section{4) CONCLUSIONS ET PERS PECTIVES}

La méthode proposée permet la détermination des ondes réfractées par une interface entre 2 milieux semi-infinis anisotropes quelconques. On montre que la connaissance des directions de propagation des ondes, issue en particulier des conditions aux limites, doit alors être complétée par celle des directions de propagation de l'énergie.

Enfin, une étude expérimentale en cours sur des échantillons monocristallins industriels nous permettra de valider notre modèle et d'en déterminer les limites [10].

D'autres part, ce formalisme est utilisé pour la résolution du problème de la propagation d'ondes dans des milieux anisotropes multicouches en incidence oblique [11].

\section{REFERENCES}

[1] Fedorov F. I., "Theory of elastic waves in crystals", Plenum, New-York, (1968)

[2] Rokhlin S.I., Bolland K., Adler L., J. Acoust. Soc. Am., 479 , 906 (1986)

[3] Ribeiro H., Thèse de doctorat, UTC (1992)

[4] Deschamps M. ,J. Acoustique, 4,269 (1991)

[5] Poirée B. ,J. Acoustique, 2, 205 (1989)

[6] Hayes M. ,Arch. Ration. Mech., 85 , 40 (1984)

[7] Rokhlin S.I., Bolland K., Adler L.,J. Appl. Phys., 1159 , 3672 (1986)

[8] Ribeiro H., de Belleval J.F., Lanceleur P., Journées GAPS US, Le Mans, (mars 1991)

[9] Ribeiro H., de Belleval J.F., Lanceleur P., article en préparation

[10] Ribeiro H., de Belleval J.F., Lanceleur P., article en préparation

[11] Potel C., de Belleval J.F., article en préparation 